\title{
NEAT: An Astrometric Mission to Detect and Characterize Nearby Habitable Planetary Systems
}

\author{
Fabien Malbet ${ }^{1}$, Antoine Crouzier ${ }^{1}$, Renaud Goullioud ${ }^{2}$, \\ Pierre-Olivier Lagage ${ }^{3}$, Alain Léger ${ }^{4}$, Mike Shao $^{2}$ and the NEAT \\ collaboration $^{5}$ \\ ${ }^{1}$ Institut de Plantologie et d'Astrophysique de Grenoble (IPAG), \\ UJF-Grenoble 1 / CNRS-INSU, UMR 5274, BP 53, F-38041 Grenoble, France \\ email: Fabien.Malbet@obs.ujf-grenoble.fr \\ ${ }^{2}$ Jet Propulsion Laboratory (JPL), California Institute of Technology \\ 4800 Oak Grove Drive, Pasadena, CA 91109, USA \\ ${ }^{3}$ Laboratoire AIM, CEA-IRFU/CNRS-INSU/Univ. Paris Diderot, CEA Saclay, UMR 7158 \\ Bat. 709, 91191 Gif-sur-Yvette Cedex, France \\ ${ }^{4}$ Institut d'Astrophysique Spatiale (IAS), Univ. Paris Sud/CNRS-INSU, UMR 8617 \\ Bat. 120-121, 91405 Orsay cedex, France \\ ${ }^{5}$ see NEAT webpage at http://neat.obs.ujf-grenoble.fr
}

\begin{abstract}
Many planets have been detected so far but very few around nearby stars that could allow characterization of their atmosphere thanks to their proximity. There are known exoplanets around less than $8.3 \%$ of the FGK stars of the Solar neighborhood $(d<20 \mathrm{pc})$ and the vast majority of them are giant planets. Within the ESA Cosmic Vision 2015-2025 plan, the scientific goal of the NEAT (Nearby Earth Astrometric Telescope) mission is to detect and characterize planetary systems around these nearby stars in an exhaustive way down to 1 Earth-mass in the habitable zone. This survey would provide the actual planetary masses, the full characterization of the orbits including their inclination, for all the components of the planetary system down to the Earth-mass limit. NEAT will continue the work performed by Hipparcos and Gaia by reaching a precision that is improved by two orders of magnitude on pointed targets compared to Gaia. We present the free-flyer concept that has been submitted to the 2010 ESA call for M3 missions with two satellites flying in formation $40 \mathrm{~m}$ apart.
\end{abstract}

Keywords. Exoplanets, Astrometry, Planet formation, High angular resolution technique

\section{Introduction}

Exoplanet research has grown explosively in the past decade, supported by improvements in observational techniques that have led to increasingly sensitive detection and characterization. Among many results, we have learned that planets are common, but their physical and orbital properties are much more diverse than originally thought.

However, it is striking that among the stars that are hosting the +900 exoplanets known so far without including the several thousands exoplanet candidates found by Kepler, only 42 FGK of such stars are located within 20 pc from the Sun, yielding to a proportion of $8.3 \%$. We know much better distant exoplanet systems than the closest ones, whereas these planetary systems will undoubtedly contain Earth-like planets whose atmosphere can be observed later spectroscopically for biosignature.

Very few nearby planetary systems will have a configuration allowing transit observation. Radial velocity has serious limitatios due to the activity of the solar-type stars 

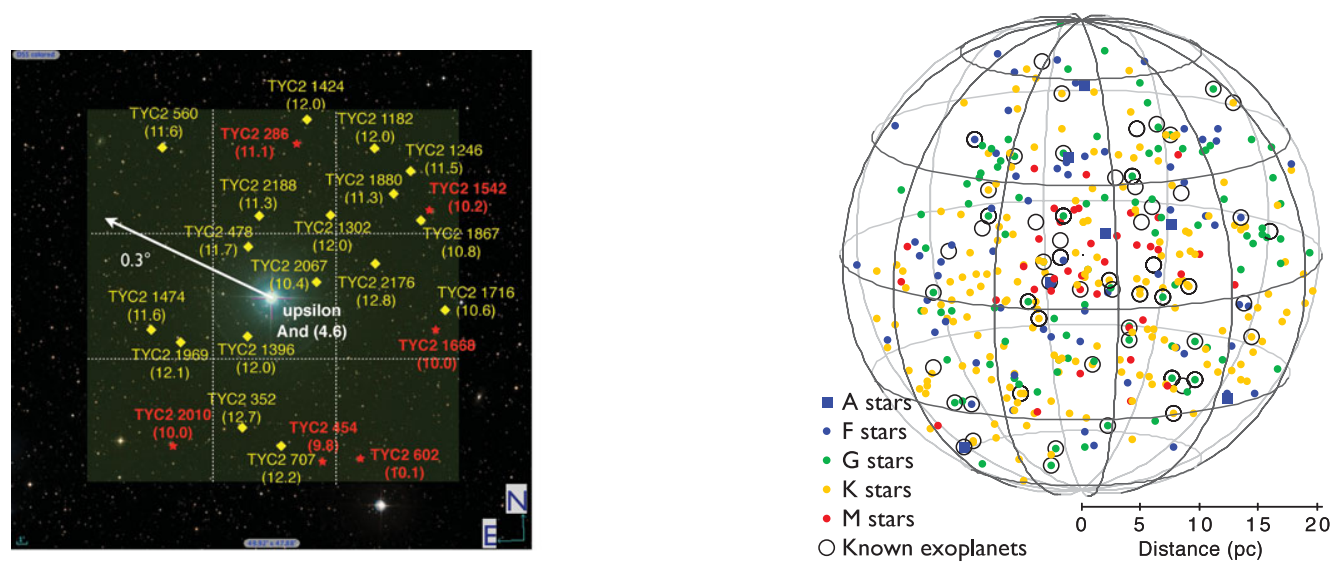

Figure 1. Left: $0.3^{\circ}$ stellar field around upsilon Andromedae, a proposed NEAT target. There are six possible reference stars in this field marked in red. Right: representation of the NEAT targets in the 3D sphere of our neighborhood $(d \leqslant 20 \mathrm{pc})$. The stars with a black circle correspond to those with known exoplanets, accounting for only $8.3 \%$ of the total number.

that will prevent a full census of the nearby systems. Although direct imaging will bring information on the radius of planets, it will be quite inefficient in detecting nearby planetary systems. Therefore astrometry appears to be the technique that will able to perform a full census of planetary systems down to the Earth-mass around solar-like stars. We present here the proposition of an high precision astrometry mission called NEAT that has been proposed for the M3 ESA call for M- Missions, which gathers about 70 scientist around Europe and worldwide.

\section{Science objectives}

\subsection{Main science questions}

The prime goal of NEAT is to detect and characterize planetary systems orbiting bright stars in the solar neighborhood that have a planetary architecture like that of our Solar System or an alternative planetary system made of Earth-mass planets. It will allow the detection around nearby stars of planets equivalent to Venus, Earth, (Mars), Jupiter, and Saturn, with orbits possibly similar to those in our Solar System. It will permit to detect and characterize the orbits and the masses of many alternate configurations, e.g. where the asteroid belt is occupied by another Earth-mass planet and no Jupiter. The NEAT mission will answer the following questions:

- What are the dynamical interactions between giant and telluric planets in a large variety of systems?

- What are the detailed processes involved in planet formation as revealed by their present configuration?

- What are the distributions of architectures of planetary systems in our neighborhood up to $\approx 15 \mathrm{pc}$ ?

- What are the masses, and orbital parameters, of telluric planets that are candidates for future direct detection and spectroscopic characterization missions?

Special emphasis will be put on planets in the Habitable Zone because this is a region of prime interest for astrobiology. Indeed orbital parameters obtained with NEAT will allow spectroscopic follow-up observations to be scheduled precisely when the configuration is the most favorable. 


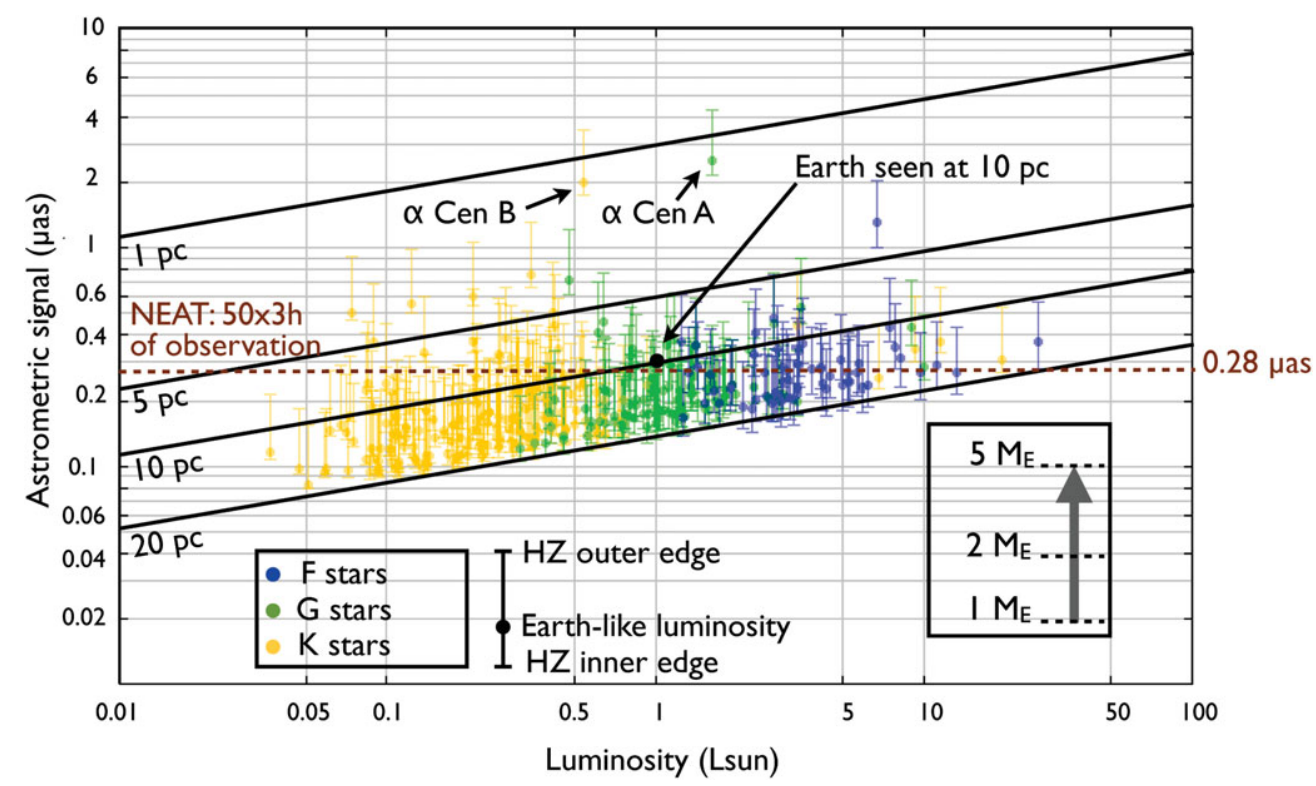

Figure 2. Astrometric signal of Earth-mass planets around nearby FGK stars. The astrometric signal is calculated for a 1 Earth-mass planet. The bars represent the inner edge and outer edges of the habitable zone of each star, the central dot is for a distance where the bolometric luminosity is equal to that of the Earth. For more massive planets the astrometric signal is shifted upward, as indicated by the arrow on the lower right.

\subsection{High-precision differential astrometry}

The principle of NEAT is to measure accurately the offset angles between a target and 6-8 distant reference stars with the aim of differentially detecting the reflex motion of the target star due to the presence of its planets. An example of a field that will be observed is shown in Fig. 1.

The output of the analysis is a comprehensive determination of the mass, orbit, and ephemeris of the different planets of the multiplanetary system (namely the 7 parameters $\left.M_{P}, P, T, e, i, \omega, \Omega\right)$, down to a given limit depending on the star characteristics, e.g. $0.5,1$ or $5 \mathrm{M}_{\oplus}$. The astrometric amplitude, $A$, of a $M_{*}$ mass star due to the reflex motion in presence of a $M_{P}$ mass planet orbiting around with a semi-major axis $a$ at a distance $D$ from the Sun is

$$
A=0.3\left(\frac{M_{P}}{1 \mathrm{M}_{\oplus}}\right)\left(\frac{a}{1 \mathrm{AU}}\right)\left(\frac{M_{*}}{1 \mathrm{M}_{\odot}}\right)^{-1}\left(\frac{D}{10 \mathrm{pc}}\right)^{-1} \mu \mathrm{as} .
$$

There are 5 parameters characterizing the star astrometric motion and 7 parameters for each orbit. A number of visits $N_{\text {visits }} \approx 50$ is sufficient to solve for the parameters of 3 to 5 planets per system, for a 5 -yr duration of the mission.

\subsection{Targets}

The spatial repartition of stars within $20 \mathrm{pc}$ is shown in Fig. 1. As for the date of May 2012 , only $8.3 \%$ are known to host exoplanets because of stellar activity problems and brightness issues. Most of the more than 800 exoplanets known in August 2012 are located further away than the first $20 \mathrm{pc}$.

A possible target list has been considered around the nearest F, G, K stars deduced from the Hipparcos 2007 catalogue. The list corresponds to an exhaustive search for 1 Earth-mass planets (resp. 5 Earth-mass planets) around K stars up to 6 pc (resp. 12 pc), 

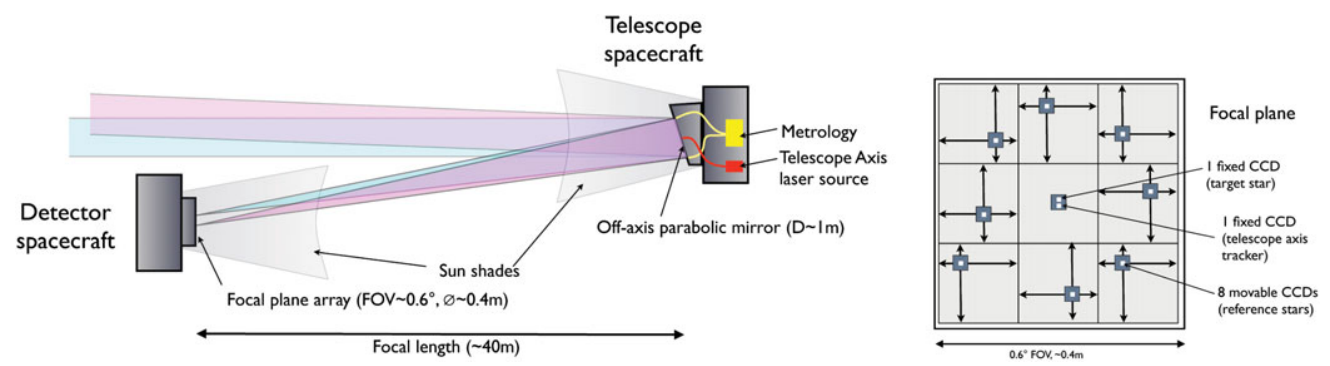

Figure 3. Proposed concept for a very high precision astrometry mission. Left: the instrument consists in two separated modules, the first one carrying the primary mirror (upper right) and the second one the detector plane (bottom left). Right: schematic layout of the focal plane. The field of view is divided in $3 \times 3$ sub-fields. Outer subfields have visible arrays which can be moved in $X$ and $Y$ directions to image the reference stars. The central field has two fixed arrays, one for the target star and one for the telescope axis tracker.

G stars up to 10 pc (resp. 17 pc), and F stars up to 14 pc (resp. 19 pc) in the entire HZ of the star, excluding spectroscopic binaries and very active stars (Fig 2).

Among the NEAT targets, $60 \%$ (118) are brighter than $V=6$ and therefore will not be investigated by Gaia because of its bright limit. So, even if some of those sources do not harbor Earth-like planets, NEAT will be contributing to the improvement of our knowledge about the neighborhood of our Solar System. In that respect, NEAT observations will not only be complementary to Gaia's ones, but NEAT data will also form a basis to improve Gaia results.

If all instrumental problems are controlled then the next obstacle to achieve the scientific objective is of astrophysical nature, the impact of stellar activity. Spots and bright structures on the stellar surface induce astrometric, photometric and RV signals. Using the Sun as a proxy, Lagrange et al. (2011) have computed the astrometric, photometric and RV variations that would be measured from an observer located $10 \mathrm{pc}$ away. It appears that the astrometric variations due to spots and bright structures are small compared to the signal of an Earth-mass planet in the HZ (Meunier et al. 2010; Makarov et al. 2009, 2010). This remains true throughout the entire solar cycle. If we consider a star 5 times more active than the active Sun, an Earth-mass planet would still be detectable even during the highest activity phases. Such activity, or lower, translates in terms of activity index $\log \left(R_{H K}^{\prime}\right) \leqslant-4.35$. Consequently, in our target list, we have kept only stars with such an index (only $4 \%$ were discarded), for which their intrinsic activity should not prevent the detection of an Earth-mass planet, even during its high activity period.

\section{NEAT concept}

Our goal is to detect the signal corresponding to the reflex motion of a Sun-like star at $10 \mathrm{pc}$ due to an Earth-mass planet in its HZ, with an equivalent final SNR of 6 . That astrometric signal is $0.3 \mu$ as. The required end-of-mission noise floor is $0.05 \mu$ as, over 100 times lower than Gaia's best precision which is $7 \mu$ as. More technical information can be found in Malbet et al. $(2012 \mathrm{a}, \mathrm{b})$.

\subsection{Instrumental concept}

The concept of the proposed mission is sketched in Fig. 3 and consists of a primary mirror - an off-axis parabolic 1-m mirror - a focal plane $40 \mathrm{~m}$ away, and metrology calibration sources. The large distance between the primary optical surface and the focal plane can 


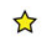

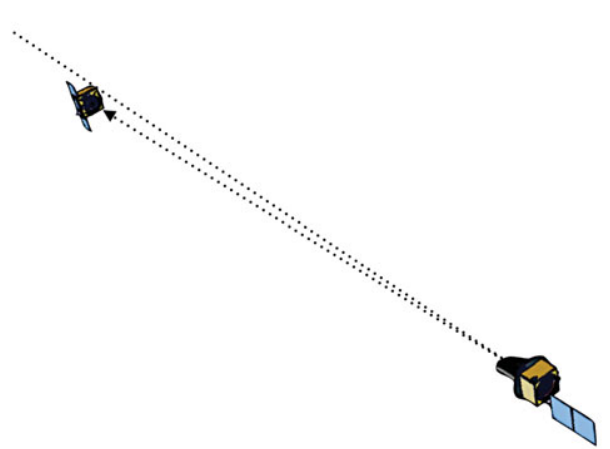

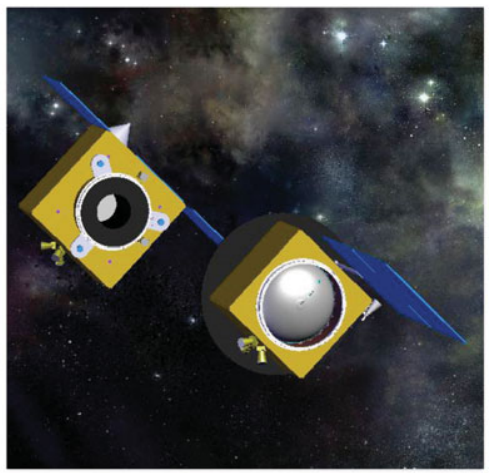

Figure 4. Left: NEAT spacecraft in operation with the two satellites separated by $40 \mathrm{~m}$. Right: closer external view of the two satellites.

be implemented as two spacecraft flying in formation, or a long deployed boom. The focal plane with the detectors having a field of view of $0.6^{\circ}$ is shown in the right part Fig. 3. It has a geometrical extent of $0.4 \mathrm{~m} \times 0.4 \mathrm{~m}$. The focal plane is composed of eight $512 \times 512$ visible CCDs located each one on an $X Y$ translation stage while the central two CCDs are fixed in position. The CCD pixels are $10 \mu \mathrm{m}$ in size.

The principle of the measurement is to point the spacecraft so that the target star, which is usually brighter $(R \leqslant 6)$ than the reference stars $(R \leqslant 11)$, is located on the axis of the telescope and at the center of the central CCD. Then the 8 other CCDs are moved to center each of the reference stars on one of them. To measure the distance between the stars, we use a metrology calibration system that is launched from the telescope spacecraft and that feeds several optical fibers (4 or more) located at the edge of the mirror. The fibers illuminate the focal plane and form Young's fringes detected simultaneously by all CCDs. The fringes have their optical wavelengths modulated by acoustic optical modulators that are accurately shifted by $10 \mathrm{~Hz}$, from one fiber to the other so that fringes move over the CCDs (Nemati et al. 2011) or by phase modulator (Crouzier et al. 2012). These fringes allow us to solve for the $X Y Z$ position of each CCD. An additional benefit from the dynamic fringes on the CCDs is to measure the QE of the pixels (inter- and intra-pixel dependence). The CCDs are read at $50 \mathrm{~Hz}$ providing many frames that will yield high accuracy.

With such a concept, the mission performance would be similar to, and even more favorable for exoplanets, than what was proposed for SIM-Lite with 5 years of operation, but at the price of giving up all-sky astrometry and the corresponding science objectives.

\subsection{Mission and spacecraft requirements}

The objectives of the NEAT mission require to perform acquisitions over a large number of targets during the mission timeline, associated to a $40 \mathrm{~m}$ focal length telescope satellite. The solution proposed for the ESA M3 mission was to use two satellites in formation flying. The L2 orbit is the preferred orbit, as it allows best formation flying performance and is particularly smooth in terms of environment. The Soyuz launch, proposed as a reference for medium class missions, offers satisfying performance both in terms of mass and volume. At L2, the solar pressure is the main disturbance for formation flying control.

The two satellites are launched in a stacked configuration using a Soyuz ST launcher and are deployed after launch in order to individually cruise to their operational Lissajous orbit. Acquisition sequences will alternate with reconfigurations, during which the Telescope Satellite will use its large hydrazine propulsion system to move around the 
Focal Plane Satellite and to point at any specified star. At the approach of the correct configuration, the Focal Plane Satellite will use a cold gas $\mu$-propulsion system for fine relative motion acquisition.

An alternative mission concept would consist of a single spacecraft with an ADAMlike deployable boom (from ATK-Able engineering) that connects the telescope and the focal plane modules. The preliminary investigation made by CNES identified no showstoppers for this option. The use of dampers on the boom structure allows damping at a level of $10 \%$ of the oscillations. The main worry concerns retargeting, which requires large reaction wheels or control momentum gyroscopes (CMGs) on the spacecraft due to the important inertia but propellers could be added at the boom end.

\section{Perspectives}

In the Cosmic Vision plan for 2015-2025, the community in Europe has identified the question "What are the conditions for planet formation?", and has recommended ultra high precise astrometry as a key technique to explore our solar-like neighbors.

We believe that there is a place for a mission like NEAT in future space programs, that is to say, a mission that is capable of detecting and characterizing planetary systems orbiting bright stars in the solar neighborhood that have a planetary architecture like that of our Solar System or an alternative planetary system partly composed of Earthmass planets. These stars visible with the naked eye or simple binoculars, if found to host Earth-mass planets, will change humanity's view of the night sky.

\section{Acknowledgements}

This work has benefited support from the Centre National des Études Spatiales (CNES), the Jet Propulsion Laboratory (JPL), Thales Alenia Space (TAS), Swedish Space Corporation (SSC) and the Labex OSUG@2020. AC PhD fellowship is funded by CNES and TAS.

\section{References}

Crouzier, A., Malbet, F., Preis, O., et al. 2012, in SPIE, Vol. 8445, article id. 84451P

Lagrange, A., Meunier, N., Desort, M., \& Malbet, F. 2011, A\&A, 528, L9

Makarov, V. V., Beichman, C. A., Catanzarite, J. H., et al. 2009, ApJ, 707, L73

Makarov, V. V., Parker, D., \& Ulrich, R. K. 2010, ApJ, 717, 1202

Malbet, F., Goullioud, R., Lagage, P.-O., et al. 2012a, in SPIE, Vol. 8442, article id. 84420J

Malbet, F., Léger, A., Shao, M., et al. 2012b, Experimental Astronomy, 34, 385

Meunier, N., Lagrange, A., \& Desort, M. 2010, A\&A, 519, A66

Nemati, B., Shao, M., Zhai, C., et al. 2011, in SPIE, Vol. 8151, E28 